\title{
Colour Associated Thermal Impacts: Manifestation and Contributing Factors with Reference to Red and Blue
}

\author{
Hettiarachchi. A A. ${ }^{1}$ \& De Silva. T.K.N.P ${ }^{2}$ \\ 1. Department of Architecture, \\ University of Moratuwa, Sri Lanka \\ 2. Department of Architecture, \\ anishkah@uom.lk \\ University of Moratuwa, Sri Lanka \\ tknpdesilva@gmail.com
}

\begin{abstract}
Theory of colour - a long-established tradition of artists - differentiates between the impacts of warm and cool colours respectfully on human beings. The lack of scientific and statistically substantiated knowledge on aforesaid colour associated thermal perception (CTP) deems to hinder its optimum integration in creating responsive built environments. This investigation seeks scientific explanation on manifestation and contributing factors of CTP with reference to a warm colour (red-RTP) and a cool colour (blue-BTP). The hypothesised potentials of CTP to manifest as a psychological response, a biological response, a factor altering core body temperature or as an actual thermal sensation caused via the human skin (due to heat radiation emitted by coloured surfaces) were tested. A crossover experiment was executed with a sample of healthy male undergraduates $(\mathrm{n}=111)$ selected via stratified simple random sampling (age 19-30), under controlled laboratory conditions. Substantiating colour theory, subjects demonstrated a propensity to perceive red as warm/hot (64.2\%) and blue as cool/cold (59.3\%). As revealed by logistic regression, CTP neither manifests due to a fluctuation in core body temperature nor as an actual thermal sensation. It is a psychological response characterised by common as well as colour-specific factors. Accordingly, thermal impacts of colour could be integrated in indoor environments to psychologically manipulate the perceived thermal environment against the real thermal condition to achieve the desirable thermal milieu. This novel knowledge may provide insights for Architects, interior designers, relevant authorities and organisations to develop strategies incorporating thermal impacts of colour to achieve low-energy design.
\end{abstract}

Keywords: Colour associated thermal perception, Infrared thermal monitoring, Likert Scale, Temporal artery temperature.

\section{Introduction}

Focusing on the imperative role of colour in creating environments responsive for human behaviour, the current study accentuates that a colour scheme, while being pleasing aesthetically, should aptly contribute to the appropriate milieu corresponding to the feelings, emotions, expressions and behavioral patterns associated with the intended activities of any built space. The human responses to colour which could be integrated in creating responsive environments are threefold.

Colour is an expressive agent according to Rihlama (1999), which can convey ideas, concepts, signs, symbols, meanings and messages associated with the intended ambience of built spaces. Colours are found to alter perceived dimensions and properties of a space; height, width, depth, proportions, weight, temperature, stability and smell (Mahnke, 1996). Most notably, colour is a psychophysiological agent which could trigger corresponding emotional and behavioural responses within humans in built spaces (Mahnke, 1996; Rihlama, 1999). Amidst the diverse colour associated human responses, the current study focuses on a less investigated and less utilized yet, an extremely important association; Colour associated Thermal Perception (CTP) of human beings, which is the macro research paradigm under consideration. 


\section{Background to Research}

As per Ballast (2002 cited in Daggett et al., 2008), colours have been theoretically explained in temperature terms. The theory of colour has been pioneered and has evolved through practice over the course of time by artists and painters. It has taken the form of a long-held tradition followed up to date, though not satisfactorily substantiated with scientific proof. The colour wheel, being the nucleolus of the traditional theory of colour, makes a clear division between duality of warm and cool colours.

Hypothetically, colour has the power to suggest warmth or coolness (Mahnke, 1996). The theories and principles on the properties, characteristics and effects of colour too are principally explained to be dual, based on the aforesaid warm/cool dichotomy. For instance, warm colors are identified to be stimulating and cool colours pacifying [Whitfield \&Wiltshire (1990); Stone (2001) and Ballast (2002)].

A considerable amount of research in the fields of experimental psychology, applied psychology and psychological ergonomics has been carried out on the possible influence of colours or coloured surfaces on thermal sensation and thermal comfort (Albers et al., 2013). The studies conducted by Itten (1961), Morton (1995), Mahnke (1996), Stone (2001), Hutchison (2003), Fenko, Schifferstein and Hekkert (2010) as well as Balcer (2014) have been able to witness the thermal impacts of colour, while the investigations by Bennett and Rey (1972), Pedersen, Johnson and West (1978), Greene and Bell (1980) as well as Ho and others (2014) have not been able to identify a significant association between colour and perceived temperature. The supportive evidence on the thermal effects of colours in the 20th century was found to be quite substandard. It has long been assumed that the impact of visual and thermal stimulation is interactive. Yet, this long-held belief has accumulated surprisingly little experimental support (Candas and Dufour, 2005). According to the literature reviewed, scholars who have attempted thus far in generalizing CTP have yielded controversial outcomes. Even though there are many hypothetical explanations, there is a significant lack of scientific investigation conducted seeking the logic, or rather, the justification behind manifestation of CTP and its significant parameters.

\section{Research Problem:}

According to Gage (1995), there are numerous unresolved problems associated with habits and behavior induced by thermal impacts of colour, which has always been a matter for debate and puzzlement in colour specification. The lack of recently conducted empirical research supported with statistically-validated evidences on CTP and the nature of its manifestation may have contributed to such controversy, hindering the utilization of CTP to create responsive built environments. The current study intends to investigate the nature of manifestation and the significant parameters of CTP.

\section{Research Objectives:}

The investigation is aimed at providing empirical evidence to the thermal impacts of colour, and to reveal the basis behind the manifestation as well as statistically significant factors of CTP with reference to a warm colour; red (RTP) and a cool colour; blue (BTP).

\section{Contributions}

Each human activity demands a corresponding comfortable thermal ambience characterized by the nature and the pace of existence for its optimum performance. For instance, class room temperature is found to have an impact on the learning process [Wargocki and Wyon (2006); Fisher (2001)]. Holmberg and Wyon (1969) revealed that students' reading comprehension and speed were reduced during elevated classroom temperature conditions. As revealed by Stone (2001), reading task was found to be lowered in a red environment. It is suggestive that red being the predominantly warm colour in the colour wheel, has caused similar impacts on reading. Setting up this thermal ambience is vital in creating environments responsive to the intended human behavior. Accordingly, comprehending the nature and parameters of 
colour associated thermal impacts will enable strategic integration of colours to manipulate the thermal environment responsive to human activities in an optimal manner.

\section{Theoretical Framework: Review of Literature}

Colour is a visual stimulus in the form of electromagnetic energy recognized to be perceived by the visual sense of humans. The eye, particularly by the photo receptive corns in the retina facilitates the visual perception process (Rihlama, 1999). Gardner (1992) defines visual perception as the ability of human brain to understand, make sense and interpret the sensory stimulus of the things seen by the eye. Preceiving thermal stimuli is firmly established to take place via the human skin, significantly by the thermal sensitive skin receptors; Ruffini's endings, Krause end bulbs (Hedge, 2008). The possibilities as to how colour, which is a visual stimulus could be perceived in thermal terms is the question under consideration. Carefully synthesizing the available literature from several disciplines, a comprehensive explanation for the nature and manifestation of CTP is summed up below.

It was assumed that a clear distinction between the phenomenon of CTP and colour temperature (black body theory) prevails. Red occurs at lower temperatures while blue occurs at higher temperatures when referring to colour temperature based on black body theory. This in fact, is the extreme opposite of the common cultural association of colour in thermal terms, which views red as hot and blue as cold [Gage, (1995); George (2008)]. 'HueHeat Hypothesis' as explained by physicists was distinguished as the most discussed and frequently studied potential explanation for CTP. The hue-heat hypothesis puts forward the idea that light waves with long wavelengths at the red end of the spectrum are felt as warm, and those at the blue end with shorter wavelengths are felt as being cool [Landgrebe et al., (2008); Michael et al., (2010); Kanaya, Matsushima and Yokosawa (2012)]. In view of this, the possibility of human skin cells and/or domains in the human eye to perceive colour in the form of heat is proposed. Based on the colour experience pyramid of Mahnke (1996), colour associated thermal impacts can be argued to be a learnt, subjective psychological response, while a part is played by the universal, archetypal associations which are common to all. Another strong theory in literature identifies CTP to be a biologically fixed bodily reaction caused due to altered core body temperature correlated with the corresponding frequency/ wavelength of each colour [Mahnke (1996); Kopacz (2003); Cornelissen and Knoop, (2012)]. Another supposition is that CTP is a synesthetic response uniting the visual and thermal senses, which is not yet substantiated and thus, inconclusive [Marks (1978); Cytowic (1989,1993); and Mahnke (1996)]. The principles of colour therapy put forward the opinion that colour energy is capable of altering core body temperature of human beings via the fixed metaphysical association between colour energy and human energy field; consequently altering their thermal perception as well [Farser (2011); Manuel (2011); Lung (2011)].

Accordingly, suppositions found in literature to explain the CTP are many. Scrutinizing and synthesising the aforementioned suppositions, three hypotheses were arrived at. These demarcate the scope and limitations of the study which set the baseline in formulating the research design; seeking scientific justification for CTP.

\section{Hypotheses}

Hypothesis 1: CTP as a Psychological Response

Possibility of CTP to be a learnt subjective psychological reaction which is unique from person to person (molded by socio, cultural, religious influences and mannerisms), and the ability of CTP to manifest as a fixed innate psychological reaction where the humans react in a universal pattern (based on primordial associations), was questioned here.

Hypothesis 2: CTP as a Fixed Biological Response; an Alteration in Core Body Temperature.

The possibility of colour stimuli triggering an actual change in the core body temperature 
(as a part of the established biological and physiological responses to colour), enabling one to perceive red as warm and blue as cool in general terms, was questioned here.

\section{Hypothesis 3: CTP as an Actual Thermal Sensation Caused due to Heat Radiation Emitted Via the Coloured Surface.}

The manifestation of CTP due to the distinctive surface temperature generated by applied colour is considered here. It is assumed that the properties of the colour such as the reflective value, absorption value and emissivity may contribute to differences in the temperature of the colored surface; consequently causing an actual thermal sensation pertaining to differences in CTP. There may be other theories and philosophies that could be used to explain CTP. For instance, synesthesia and metaphysical correlation could be considered. However, all these cannot be covered due the vastness of the area and considered as future research directions.

\section{Research Design}

While qualitative and quantitative methods are being mixed in several stages, the current study was executed as a mixed model research. A sample representing the undergraduate male population of Faculty of Architecture, University of Moratuwa (Sri Lanka), was selected via stratified simple random sampling representing four affiliated sub populations; departments. This sample has a rich mix of students representing a fair cross-section of the whole country and the different secondary educational streams followed, enabling the findings to be generalized to a greater extent. The sample was limited only to males to assure more validity in data as females are established to demonstrate fluctuations in the core body temperature caused by ovulation [Swedan (2001); Barron and Fehring (2005)].

A three stage thorough screening process was designed to filter the best fitting subjects from the initial sample $(\mathrm{n}=155)$, which also served as the first phase of data collection. Preliminary screening was conducted via a questionnaire survey on known medical history (selfreport), while the written consent of subjects was obtained via a letter of consent, fulfilling ethical clearance.

The second phase of screening was a general health checkup carried out by a registered medical practitioner. It was conducted to test the vision, general physical and mental health condition and most importantly, to screen the subjects having illnesses pertaining to a high or low core body temperature than the normal range. Subjects qualifying for the next level were provided with an explicit set of guidelines to follow as preparation for the final trial.

Final screening was an eligibility interview done just before entering the lab to recheck the physical and psychological fitness of the subjects. Checks on adherence to the set guidelines were made and subjects with temperatures beyond normal core body temperature were screened out. The final sample consisted of most eligible $111(\mathrm{n}=111$ with $95 \%$ confidence level and $7.5 \%$ margin for error).

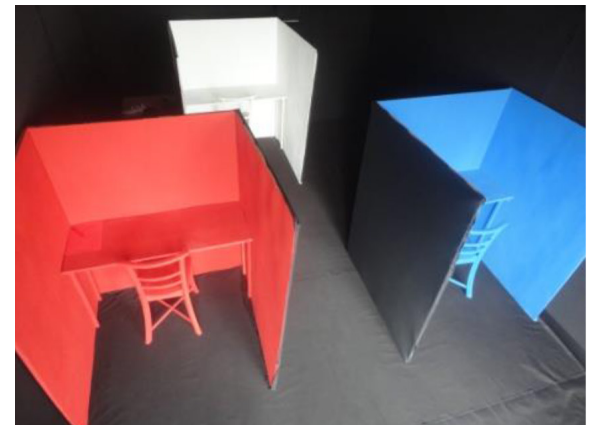

Figure 1: Colour Lab with Three Colour Workstations

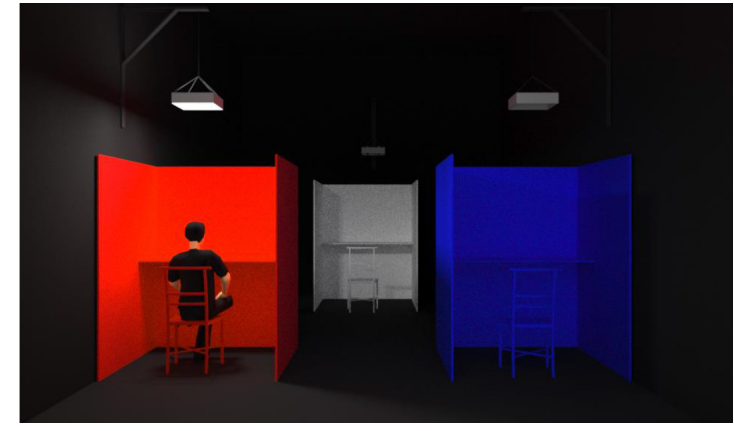

Source: Author (2014). 
Thermal perception of the subjects was tested in two coloured workstations - Red (RWS) and Blue (BWS) - positioned within controlled laboratory conditions; fixed room temperature (78.8 o F; 26o C), humidity (50\% rh), lighting level (350 lux - Asian standards) and fixed interior finishes (matt) and colour (black). Red and blue were selected in the investigation to study the associated thermal impacts with careful deliberation to have the best representation of warm and cool dual colour paradigms. The study investigated the impact of a single, flat colour with a matt finish, and did not measure any outcomes for the effect of colour combinations, finishes, textures or colour patterns as this would widen the scope of research.

A White Workstation (WWS) having identical conditions was integrated as the control and to washout carryover effects from first colour treatment. The investigation was conducted as a crossover trial having two study arms; BR Study arm (Blue treatment followed by red treatment) and RB study arm (Red treatment followed by blue treatment). Prior to first colour treatment, each subject was kept inside the WWS for 10-15 minutes until he reached his basal temperature level. Experiment started with the subject being exposed to RWS (alternately BWS) for 15 minutes. In order to washout carry over effects from the first treatment, the person in question was sent back to the WWS for another 10 minutes. Once the subject reached a constant/basal temperature level, he was exposed to the BWS (alternately RWS) for another 15 minutes. Out of 10 research subjects that underwent through the trial per a day, equal number of subjects were allocated to both study arms (RB study arm = 5 and BR study arm $=5$; in total 50-RB and 51-BR).

Colour of the Work Stations being the independent variable, was manipulated to test the impact on thermal perception; the dependent variable. All the aspects of experiment was designed to control the diverse participant and situational extraneous parameters of visual perception and thermal perception. The confounding factors beyond control of the investigation were recorded or measured and included in the regression model.

During the first phase of data collection which took place simultaneously with the screening process, a part of qualitative data associated with predictor variables [i.e. vision (Vis), level of previous exposure to colour theory and usage (Loe), religion (Rlgn), race (Rce), study stream followed for secondary education (Sec Edu), favorite colour/colours (Fvc), sensitivity to warm conditions (Sen_Warm), sensitivity to cool conditions (Sen_Cool), rate of sweating (Ros) and quantitative data, average temperature of the home town (HT temp), body mass index (BMI) and age] were recorded.

The second phase of data collection was done during the lab experiment where CTP in RWS and BWS (RTP and BTP) was rated by the subjects in a 5-pont Likert scale. Qualitative data related to predictor variables; feelings and emotions induced (R_Psy/B_Psy) and preference to colour exposure $(\mathrm{R}$ Pre/ $\mathrm{B}$ Pre) in RWS and BWS were reporte $\bar{d}$ by the subjects via a questionnaire survey.

Quantitative data related to predictor variables too were recorded. Temporal artery temperature (TAT) of subjects in RWS and BWS via a Temporal Scanner, Surface temperature of RWS (R_SFT) and BWS (B SFT) using Inferred thermal monitoring (FLIR i60 camera), outdoor temperature (OT) via a mini data logger and test time zone (TTZ) by a digital clock were also monitored.

Analysis of the data was done with the use of statistical regression. Complex Sampling Ordinal Logistic Regression Model(CSOLRM) was applied for both RTP and BTP including 18 predictor variables in order to provide a statistically significant explanation and to understand the most powerful explanatory variables.
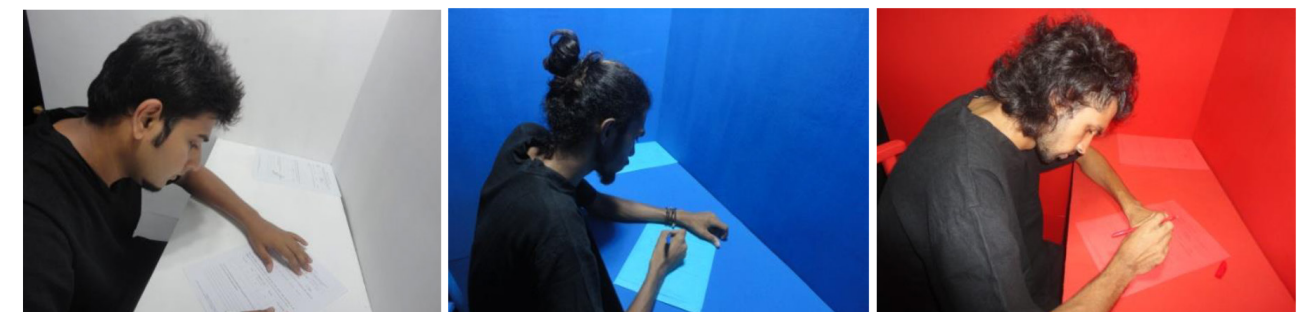

Figure 2; Research Subjects Undergoing Colour Exposure $\quad$ Source: Author (2014).

Built-environment: Sri Lanka - Research Journal of the Sri Lanka Institute of Architects / Hettiarachchi \& De Silva. 


\section{Analysis}

RTP vs BTP

\section{RTPvs BTP}

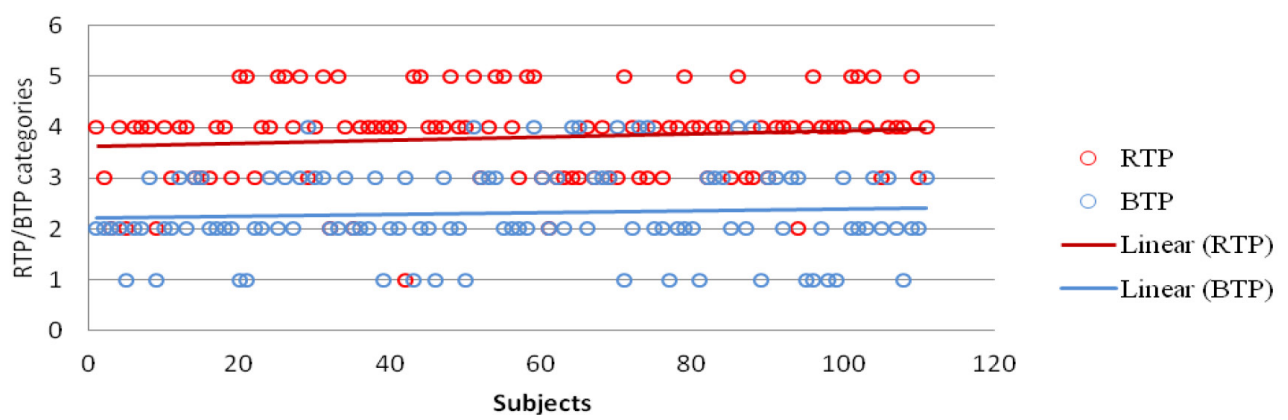

Figure 3: RTP vs. BTP

Source: Author (2014).

Substantiating thermal impacts of warm and cool colours $64.20 \%$ of the subjects perceived red as warm/ hot and $59.30 \%$ perceived blue as cool/ cold. The above graph demonstrates that the trend line of RTP values lie on a higher level than BTP. Accordingly, BTP is found to be cooler and RTP to be warmer.

\section{Comparison between RTAT and BTAT}

RTAT fluctuation: $-0.4^{\circ} \mathrm{F}-0.8^{\circ} \mathrm{F}$, Avg: $0.083^{\circ} \mathrm{F}$.

BTAT fluctuation: $-0.4^{\circ} \mathrm{F}-0.9^{\circ} \mathrm{F}$, Avg: $0.08^{\circ} \mathrm{F}$.

Diff_RTAT_Avg-WTAT basal vs Diff_BTAT_Avg - WTAT Basal

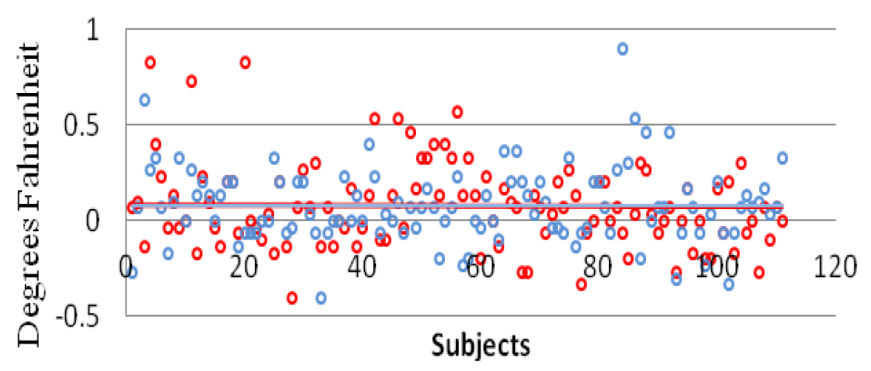

Diff_RTAT_Avg-WTAT10_1

Diff_BTAT_Avg-WTAT10_1

Figure 4 ; Body Temperature Fluctuations for Red and Blue Exposure

Source: Author (2014).

As per the scatter plot representing the average temporal artery temperature fluctuation of each subject when exposed to red and blue from the basal temperature level reached in WWS, both trend lines overlap and run horizontally. Accordingly, it is suggestive that CTP is not associated with an alteration in core body temperature.

Surface temperature of RWS vs. BWS

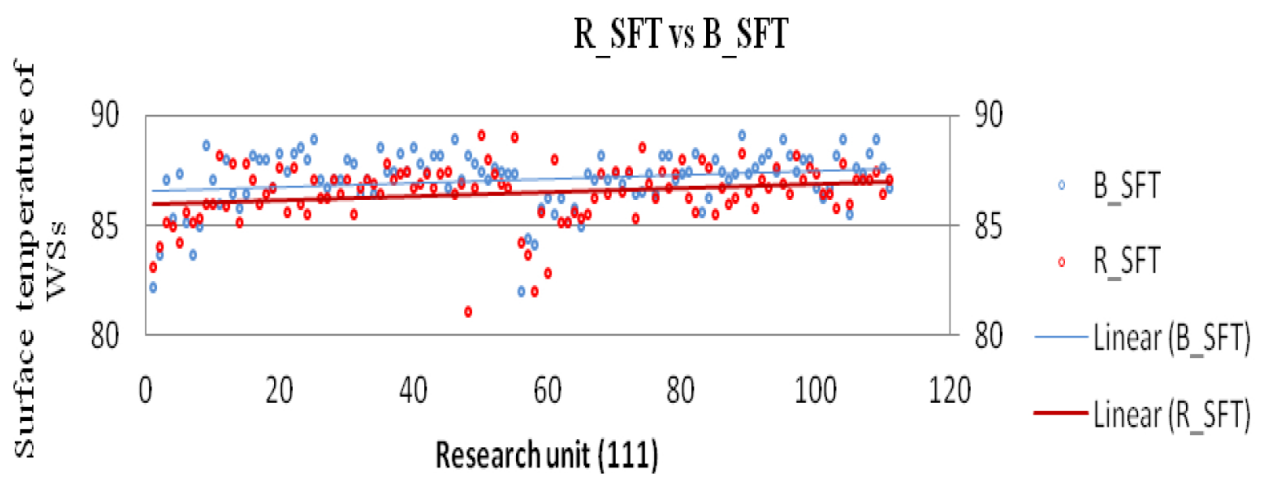

Figure 5: Surface Temperature of Red/ Blue Work Stations

Source: Author (2014) 
The temperature of blue surface (B_SFT) was found to be mildly higher than temperature of the red surface (R_SFT), suggesting the possibility of colour to emit heat/radiation, potentially playing a part in the external thermal milieu (average difference: $0.71^{\circ} \mathrm{F}$ ); even if a majority of subjects perceived BWS to be cooler and RWS as warmer. However, considering the measurement error of the camera ( $2 \%$ of the reading), observed average increase of B_SFT value $\left(0.71^{\circ} \mathrm{F}\right)$ cannot be considered as a significant increase.

\section{Complex Sampling: Ordinal Regression - RTP - Significant Model}

$\mathrm{RTP}=\mathrm{b} 0 \times \mathrm{Sec} E \mathrm{Edu}+\mathrm{b} 1 \times \mathrm{FVC}+\mathrm{b} 2 \times \mathrm{R} \operatorname{lgn}++\mathrm{b} 3$ $\mathrm{x}$ R_Psy + b4 x R_Pre

As per the test of model effects it was found that the predictors Sec Edu, FVC, Rlgn, R Psy, R_Pre are statistically significant for RTP.

\section{Complex Sampling: Ordinal Regression - BTP - Significant Model}

$\mathrm{BTP}=\mathrm{b} 0$ (Sec_Edu) $+\mathrm{b} 1$ (Age) + b2 (B_Psy) + b3 (B Pre) + b4 (B SFT)

As per the test of model effects it was found that the predictors Sec_Edu, Age, B_Psy, B Pre and B_SFT are statistically significant for BTP.

A major finding of the statistical analysis is the common significance of psychological factors for CTP. Psychological state induced by the colour exposure (R Psy / B Psy), preference to colour exposure (R_Pre / B_Pre) and preconceived learnt ideas of the secondary education stream followed (Sec Edu) dominate as commonly significant factors for both RTP and BTP. Adding to psychological factors, favorite colour (FVC) and religion (Rlgn) were identified as statistically significant for RTP. BTP was found to be affected by the subject's age. Parallel to the hunch that SFT could have an impact on RTP/BTP, it was found that B SFT is significant for BTP.

None of the considered parameters of (actual) thermal perception were identified as significant for nei-ther RTP nor BTP (other than B SFT which was found to be significant for $\bar{B}$ TP) by the statistical model, suggesting that CTP is not linked to an actual thermal sensation. Average B_SFT being $0.71^{\circ} \mathrm{F}$ higher than R_SFT signified the possibility of coloured surfaces to emit heat/radiation, yet not in an impacting level.

CSOLRmodel rejected temporal artery temperature fluctuation as being significant for both RTP and BTP.

RTP Model: Pseudo R2 = .334 (Cox and Snell); .364 (Nagelkerke)

BTP Model: Pseudo R2 $=.371$ (Cox and Snell); .405 (Nagelkerke)

In the current investigation, RTP models gave correct classification for $55.2 \%$ of the predictors studied. BTP provided classification for $58.3 \%$, suggesting a good model fit.

\section{Conclusions}

This investigation, while adding to the minimal amount of supportive empirical evidence on the dichotomous impacts of warm and cool colors on thermal perception, claims its originality for filling the long awaited research gap for an empirical explanation on the nature of CTP (with reference to its manifestation and significant parameters). It was revealed that CTP neither manifests as a result of a fluctuation in core body temperature by means of a biological reaction nor, does it demonstrate traits of an actual thermal sensation caused physically via the human skin. CTP experienced by normal sighted healthy subjects demonstrates traits to be a psychological response with combinations of learnt reactions as well as certain common connotations. Further, CTP manifests to the most part as a response to visually transmitted thermal information which are interpreted via this psychological process. Accordingly, colour is a 'non-thermal stimuli' and the associated impacts are induced psychologically. Irrespective of the real indoor thermal condition of the laboratory which was regulated at $78.8^{\circ} \mathrm{F}$, the presence of red colour in the RWS has been able to psychologically manipulate the subjects to perceive the environment as warmer while exposure to blue 
colour in BWS has psychologically induced a cooler perception.

It is recommended to extend the study further with more diverse samples to test the impacts of age, gender, religion and sociocultural implications in determining the causes of CTP.

This key new knowledge has a greater significance in architectural and interior design related solutions, which may lead the future colour applications into a different dimension (where colour works as a psychological agent to generate thermally conducive, comfortable and responsive environments sup-portive in engendering optimum human behaviour. Further, providing insight for Architects, interior designers, authorities and organisations, colour can be incorporated as an effective agent to manipulate the perceived indoor thermal environment against the real thermal condition. This may enable the inhabitants to reach comfort levels consuming comparatively less energy leading to a potential hybrid method of energy conservation.

\section{References}

Albers, F., Winzen, J. and Marggraf-Micheel, C. (2013), 'The Influence of Coloured Light in the Air-craft Cabin on Passenger Thermal Comfort', Lighting Research and Technology, 0, pp. 1-11.

Balcer, C. A. (2014) 'Visual Cues Effects on Temperature Perception', M. Sc. Thesis, Northern Michigan University.

Ballast, D. K. (2002) Interior Design Reference Manual, Belmont, CA.: Professional Pub, Inc. CA.

Barron,M. L. and Fehring, R. (2005), 'Basal Body Temperature Assessment: Is It Useful to Couples Seeking Pregnancy?', American Journal of Maternal Child Nursing, 30(5).

Bennett, C. A. and Rey, P. (1972), 'What's So Hot about Red?', Human Factors, 14(2), pp. 149-154.

Candas, V. and Dufour, A. (2005), 'Thermal Comfort: Multisensory Interactions?', Journal of Physio logical Anthropology and Applied Human Science, 24(1), pp.33-36.
Cornelissen, S. and Knoop, M. (2012), 'Lighting Design: Creating a Less Intimidating Hospital Experience', worldhealthdesign, [online]. Available at: http://www. worldhealthdesign.com/Lighting-Design. aspx [Accessed 20 February 2014].

Cytowic, R. E. (1989) Synesthesia: A Union of the Senses, New York: Springer Verlag.

Daggett, W. R., Cobble, J. E. and Gertel, S. J. (2008) Color in an Optimum Learning Environment, Rexford, NY: International Center for Leadership in Education.

Fenko, A., Schifferstein, H.N.J. and Hekkert, P. (2010), 'Looking Hot or Feeling Hot: What Determines the Product Experience of Warmth?', Materials \& Design, 31, pp. 1325-1331.

Fisher, K. (2001) Building Better Outcomes: The impact of school Infrastructure on Student Outcomes and Behavior, Canberra: Department of Education, Training and Youth Affairs.

Fraser, T. L. (2011) Body: Connection, USA: Xlibris Corporation.

Gage, J., 1995. Color and Culture. In: Lamb, T. and Bourriau, J., eds. Colour: Art \& Science, Cambridge: Cambridge University Press, 1995.

Gardner, M. F. (1992) Test of Visual-Perceptual Skills (Non-Motor): Upper Level, Tvps $n$-m:UI, Illustrated Edition, California: Psychological and Educational Publica-tions Inc.

George, C. (2008) Mastering Digital Flash Photography: The Complete Reference Guide, New York: Sterling Publishing Company.

Green, T. C. and Bell. P. A. (1980), 'Additional Considerations Concerning the Effects of 'Warm' and 'Cool' Wall Colours on Energy Conservation, Ergonomics, 23, pp. 949-954.

Hedge, A., 2008. Thermal Sensation and Thermoregulation DEA 3500 - Human Factors: Ambient Environment, lecture notes. 
[online]. Available at: http://ergo.human. cornell.edu/AHSyllabi/DEA35002013. html [Accessed 11 December 2013].

Ho, H., Iwai, D. and Nishida, S. (2014), 'Combining Colour and Temperature: A Blue Object is More Likely to be Judged as Warm Than a Red Object', Scientific Reports, 4.

Holmberg, I. and Wyon, D. P. (1969), 'The Dependence of Performance in School on Classroom Temperature', Educational and Psychological Interactions, 31.

Hutchison, E. (2003) Dimensions of human behavior; Person and Environment, CA: Thousand Oaks, Sage Publications.

Itten. J. (1961) The Art of Colour: The Subjective Experience and Objective Rationale of Colour, New York: Reinhold Publications Co.

Kanaya, S., Matsushima, Y. and Yokosawa, K. (2012), 'Does Seeing Ice

Really Feel Cold? Visual-Thermal Interaction under an Illusory

Body-Ownership', PLoS ONE, 7(11), [online]. Available at: https://journals.plos. org/plosone/article?id=10.1371/journal. pone.0047293[Accessed 10 January 2014].

Kopacz, J. (2003) Colour in Three-Dimensional Design, New York: McGraw-Hill.

Landgrebe, M., Nyuyki-Dufe, K., Frank, E. and Steffens, T. (2008), 'Effects of Colour Exposure on Auditory and Somatosensory Perception - Hints for Cross-modal Plasticity', Neuro Endocrinol, 29, pp. 518-521.

Lung,H. (2011) Ultimate Mind Control: Asian Arts of Mental Domination, New York: Kensington Publishing Corp.

Mahnke, F. H. (1996) Colour Environment Human Response, New York: Wiley \& Sons.

Manuel, E. (2011) Finding Happiness: Stress Relief for a Happier Life, CAL: Metamind Publishing.
Marks, L.E. (1978) The Unity of the Senses: Interrelations Among the Modalities, New York: Academic Press.

Michael, G. A., Galich, H., Relland, S. and Prud'hon, S. (2010), 'Hot Colors: the Nature and Specificity of Color-induced Nasal Thermal Sensations', Behavioral Brain Re-search, 207, pp. 418-428.

Morton, J. L. (1995), 'Color \& Energy Matters', colormatters, [online]. Available at: http:// www.colormatters.com/color- and- science/ color-and-energy-matters [Accessed 13 March 2014].

Pedersen, D. M., Johnson, M. and West, J. H. (1978), 'Effects of Room Hue on Ratings of Self, Other and Environment', Perceptual and Motor Skills, 46, pp. 403-410.

Rihlama, S. (1999) Colour World, Helsinki: The Finnish Building Centre Ltd.

Stone, N. (2001), 'Designing Effective Study Environments', Journal of Environmen-tal Psychology, 21(2), pp. 179190.

Swedan, N. G. (2001) Women's Sports Medicine and Rehabilitation, Pennsylvania: Lippincott Williams \& Wilkins.

Wargocki, P. and Wyon, D. P. (2006), 'Research Report on Effects of HVAC on Stu-dent Performance, ASHRAE Journal, 48 (10), pp. 23-28.

\section{List of Figures}

Fig 1: Colour Lab with Three Colour Workstations Source: Author (2014).

Fig 2: Research Subjects Undergoing Colour Exposure Source: Author (2014).

Figure 3: RTP vs. BTP

Source: Author (2014).

Figure 4: Body Temperature Fluctuations for Red and Blue Exposure Source: Author (2014).

Figure 5: Surface Temperature of Red/ Blue Work Stations Source: Author (2014). 